\title{
Wide Refractive Index Detection Range Surface Plasmon Resonance Sensor Based On D-shaped Photonic Crystal Fiber
}

Honggang Pan

Tianjin University of Technology

Fei Pan

Tianjin University of Technology

Ailing Zhang ( $\square$ alzhang@email.tjut.edu.cn )

Tianjin University of Technology

Chuanbo Cao

Tianjin University of Technology

Fengjun Xue

Wuhan Running Education Research Institute

\section{Research Article}

Keywords: Surface plasmon resonance, photonic crystal fiber, refractive index detection, sensor.

Posted Date: February 11th, 2022

DOI: https://doi.org/10.21203/rs.3.rs-1126729/v1

License: (9) This work is licensed under a Creative Commons Attribution 4.0 International License. Read Full License 


\title{
Wide refractive index detection range surface plasmon resonance sensor based on D-shaped photonic crystal fiber
}

\author{
Honggang Pan a, Fei Pan a , Ailing Zhanga ${ }^{*}$, Chuanbo Cao ${ }^{\text {a }}$, Fengjun Xue ${ }^{b}$ \\ ${ }^{a}$ Engineering Research Center of Communication Devices and Technology, Ministry of Education, Tianjin Key \\ Laboratory of Film Electronic and Communication Devices, School of Integrated Circuit Science and Engineering, \\ Tianjin University of Technology, Tianjin 300384, China \\ ${ }^{\mathrm{b}}$ Wuhan Running Education Research Institute, Wuhan 430070, China \\ *Address all correspondence to Ailing Zhang, E-mail: alzhang@email.tjut.edu.cn
}

\begin{abstract}
We propose a surface plasmon resonance (SPR) refractive index (RI) sensor based on a novel D-shaped photonic crystal fiber. We used an open air hole coated with gold film to improve SPR effect. The effects of structural parameters on resonance spectrum were analyzed by finite element method (FEM). Three SPR peaks were obtained in the wavelength range of $800 \mathrm{~nm}-1300 \mathrm{~nm}$. The simulation results show that the refractive index detection range of the sensor is 1.26-1.38. The maximum sensitivity is $5626.86 \mathrm{~nm} / \mathrm{RIU}$ and the maximum resolution is $1.78 \times 10^{-5} \mathrm{RIU}$ at the refractive index of 1.38 . The sensor uses photonic crystal fiber with the same air holes diameter. Compared with other D-shaped structures, our sensor can measure larger RI ranges of the analyte.
\end{abstract}

Index Terms: Surface plasmon resonance, photonic crystal fiber, refractive index detection, sensor.

\section{Introduction}

In the last few decades, surface plasmon resonance (SPR) has attracted extensive attention and research due to its unique advantages in the fields of chemical sensing, biomedical diagnosis and biochemical reaction detection[1-5]. Resonance occurs when the wavelength of photons of the incoming electromagnetic wave matches with the wavelength of surface electrons. Under the condition of resonance, an unknown analyte with different refractive index (RI) can be detected through observing the variation of loss peak and corresponding resonance wavelength. When SPR effect was applied to sensing, SPR sensor was based on Kretschmann [6] and Otto [7]. However, their configuration is complicated and lack mobility. Therefore, the optical fiber sensor made of optical fiber has entered the field of vision of researchers.[8]. In recent years, SPR sensors based on photonic crystal fiber (PCF) have attracted much attention due to its flexible structure and other structural characteristics[9]. In general, SPR sensors based on PCF can be divided into two categories. The first type is the selective filling metal and liquid analytes into pores, which has been widely reported[10-13]. However, this type of sensor can not realize real-time sensing. The reason is that the air holes need to be refilled when the RI of analyte changes. In addition, it is extremely difficult to coat or fill the micro holes of the sensor with metal foil or infiltrate analytes. The emergence of the second structure (D-shaped PCF-SPR sensor) solves the limitations of the first structure. The D- 
shaped PCF-SPR sensor places metals and analytes in the outer layer of the photonic crystal fiber to avoid the complex operation of filling holes. C. Liu et al. designed a SPR sensor with two open air holes based on PCF[14]. The sensor is designed to detect low refractive indexes between 1.23 and 1.29 with the operation wavelength in mid-infrared region. The spectral sensitivity is $11000 \mathrm{~nm} / \mathrm{RIU}$ and a maximum resolution of $7.69 \times 10^{-6} \mathrm{RIU}$ can be obtained. X. Chen et al. deposited a SPR sensor with two open-ring channels based on PCF[15]. Simulation results show that the sensing of the sensor ranges from 1.20 to 1.29 . The maximum spectral sensitivity of $11055 \mathrm{~nm} / \mathrm{RIU}$ and high resolution of $9.05 \times 10^{-6}$ RIU can be obtained at 1.29. A. K. Pathak et al. proposed and analyzed a concave refractive index sensor based on surface plasmon resonance[16]. Results exhibit a high sensitivity of $9314.28 \mathrm{~nm} / \mathrm{RIU}$, respectively for the RI varying between 1.33 to 1.38 with the resolution of $1.073 \times 10^{-5}$ RIU. S. Wang et al. investigated a SPR refractive index sensor based on a Dshaped Hi-Bi PCF [17]. Simulation results indicate that the high sensitivity of $8920 \mathrm{~nm} / \mathrm{RIU}$, respectively for the RI varying between 1.33 to 1.39. In previous studies, the refractive index detection range of most D-type SPR sensors is small. Meanwhile, a large number of sensors used PCF with different air hole diameters, which increases the difficulty in sensor fabrication.

In this work, we designed a surface plasmon resonance sensor based on a novel D-shaped photonic crystal fiber structure. Deposition of gold film in the open air hole can not only provide channels for analytes, but also enhance the resonance effect. Three SPR peaks were obtained in the wavelength range of $800 \mathrm{~nm}-1300 \mathrm{~nm}$. Simulation results show that the refractive index sensor has a broad measurement range from 1.26 to 1.38 and that the maximum sensitivity can reach to $5626.86 \mathrm{~nm} / \mathrm{RIU}$. They also show that the refractive index resolution is $1.78 \times 10^{-5} \mathrm{RIU}$.

\section{Sensor design and numerical modeling}

The Schematic illustration of our proposed D-shaped PCF is shown in Fig. 1. This structure is composed of two layers regular hexagonal air holes with the same diameter, and the diameter $d$ of the air holes is $1.4 \mu \mathrm{m}$. The hole-to-hole pitch is $\Lambda=3.1 \mu \mathrm{m}$, and $H=3.5 \mu \mathrm{m}$ is the distance between the D-shaped section and the fiber core. In order to excite the SPR, a thin gold film with thickness $t=45 \mathrm{~nm}$ is deposited on the inner surface of the open air hole analyte channel.

In this fiber, the D-shaped structure can be fabricated using stack-and-draw method and side polishing technique, and the gold film can be coated with a chemical deposition technique[18]. In our simulation, the background material of the proposed PCF is fused silica, and its refractive index is determined by Sellmeier equation[19]. The dielectric constant of gold is described by the Drude-Lorentz model [20].

The confinement loss of this sensor can be expressed as[21]:

$$
\alpha_{\text {loss }}=8.686 \times \frac{2 \pi}{\lambda} \operatorname{Im}\left(n_{\text {eff }}\right) \times 10^{4}(\mathrm{~dB} / \mathrm{cm})
$$

where $\lambda$ is the wavelength of incident light, and $\operatorname{Im}\left(n_{\text {eff }}\right)$ is the imaginary part of the effective refractive index. As an important parameter to evaluate the sensing performance, the spectrum sensitivity is expressed as:

$$
S(\lambda)=\frac{\Delta \lambda_{\text {peak }}}{\Delta n_{a}}(\mathrm{~nm} / \mathrm{RIU})
$$

where $\Delta \lambda_{\text {peak }}$ represents the resonance wavelength shift and $\Delta n_{a}$ denotes the change in the analyte RI. The refractive index of resolution is defined as: 


$$
R=\Delta n_{a} \cdot \Delta \lambda_{\text {min }} / \Delta \lambda_{\text {peak }}
$$

where $\Delta \lambda_{\min }$ is the minimum spectral resolution assumed to be $0.1 \mathrm{~nm}$.

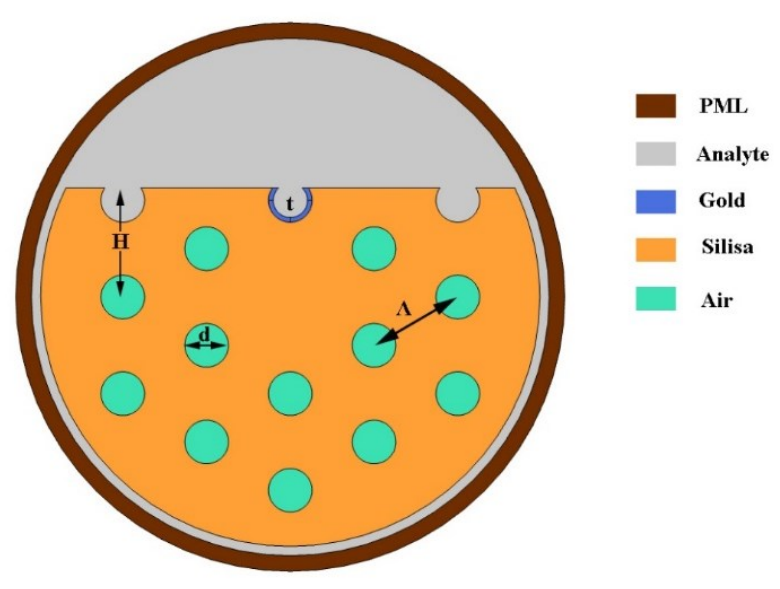

Fig. 1. Schematic illustration of the proposed D-shaped PCF sensor.

For the purpose of accurate calculation, a perfect matched layer (PML) is introduced in the calculation zone edges to absorb radiation energy. The simulation process is accomplished by the commercial finite element method solverCOMSOL Multiphysics.

\section{Results and discussion}

Fig. 2 (a) shows the three peaks in the wavelength range of $800 \mathrm{~nm}-1300 \mathrm{~nm}$ when analyte RI $n_{a}=1.3$. Fig. 2 (b) (c) and (d) illustrate the dispersion relations of SPP mode and fundamental mode of the three peaks. The inserts in Fig. 2 are the electric field distributions both of the two modes at specific wavelengths. The characteristic of SPR is that the real part of the effective index of the fundamental mode and surface plasmon-polaritons (SPP) mode coincides, and a peak appears in the imaginary part of the effective index. The maximum energy transfers from the fundamental mode to the SPP mode at the resonant wavelength. These three resonance peaks are the result of the interaction between different modes. Fig. 2 (b) shows that the resonance between x-polarization and high-order SPP mode occurs at $894 \mathrm{~nm}$. The resonance between y-polarization and high-order SPP mode occurs at $1026 \mathrm{~nm}$ can be observed in Fig. 2 (c). It can be seen from Fig. 2 (d) that the resonance between x-polarization and low-order SPP mode occurs at $1238 \mathrm{~nm}$. It should be noted that there is a fourth resonant peak at a longer wavelength, which is caused by the resonance between y- polarization and low-order SPP mode. However, the fourth resonant peak has a longer wavelength span and is not suitable for sensing research. According to the intensity and FWHM (Full width at half maxima) of the resonance peaks, we choose peak 3 as the research object.

In order to obtain the loss spectrums with large peak value and small FWHM, the effects of air hole diameter $\mathrm{d}$ and hole-to-hole pitch $\Lambda$ on the loss spectrums were studied. Fig. 3(a) displays the change of loss spectrums and resonance wavelength when the air hole diameter is $1.3 \mu \mathrm{m}, 1.4 \mu \mathrm{m}$ and $1.5 \mu \mathrm{m}$ respectively. Other parameters are fixed at $\Lambda=3.1 \mu \mathrm{m}$, $H=3.5 \mu \mathrm{m}, t=45 \mathrm{~nm}$ and $n_{a}=1.3$. As shown in Fig. 3(a), with the increase of air hole diameter, the loss peak of the sensor 
moves to the longer wavelength direction. The reason is that larger sized holes lead to a smaller $\operatorname{Re}\left(\mathrm{n}_{\text {eff }}\right)$ value of fundamental mode. Eventually, the phase matching point will move towards the long wavelength. The air hole diameter of $1.4 \mu \mathrm{m}$ has a sharper resonance peak, so it can be used as the best air hole diameter.
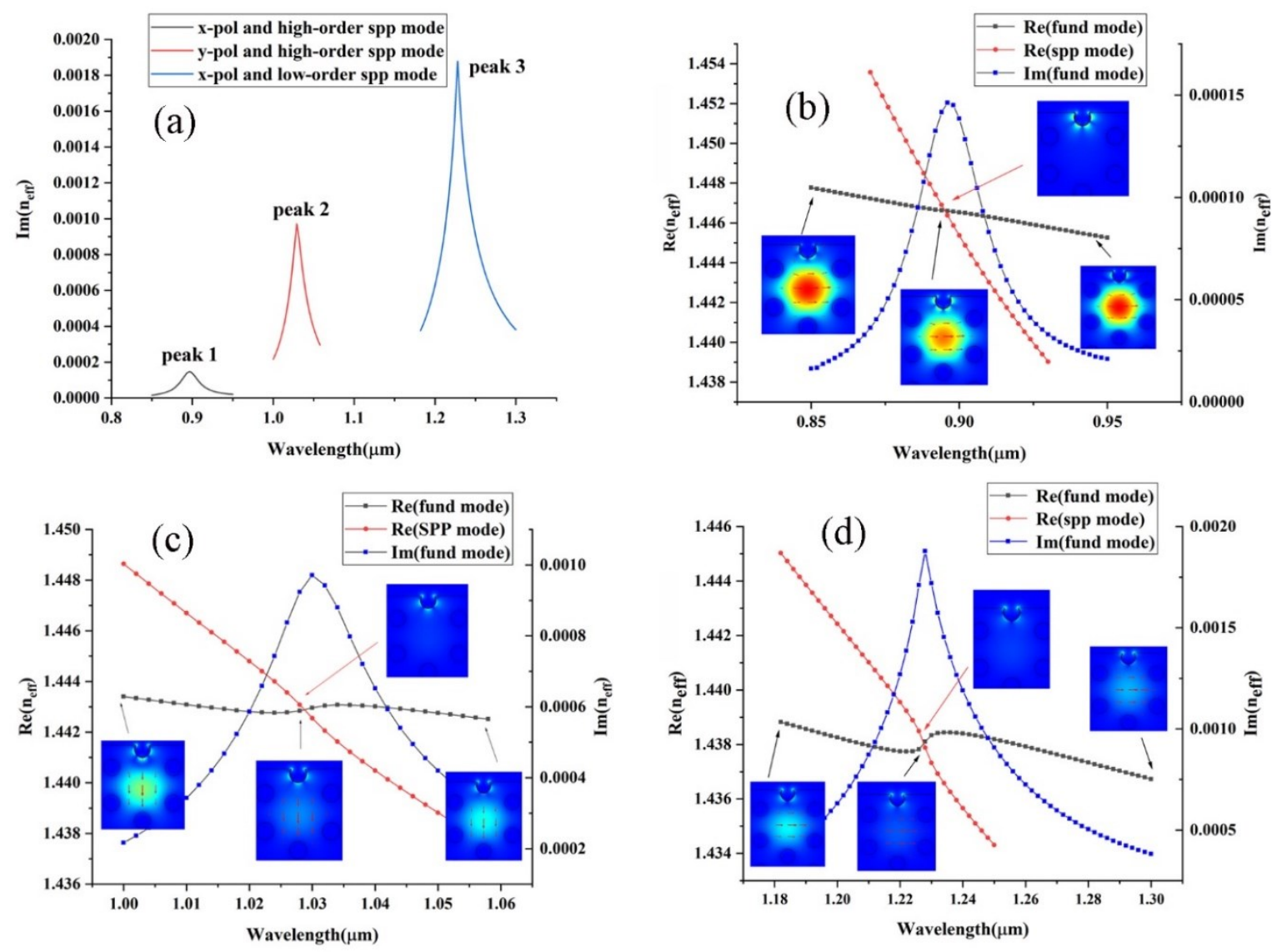

Fig. 2. Three resonance peaks excited by sensing structure (a), dispersion relation of fundamental core mode, SPP mode, and loss spectra (b-d).

Fig. 3(b) exhibits the loss spectra variations for different degrees of gold film thickness t ranging from $40 \mathrm{~nm}$ to 50 $\mathrm{nm}$. As is shown, the loss spectrum moves towards longer wavelength with $t$ decreasing from $50 \mathrm{~nm}$ to $40 \mathrm{~nm}$. The reason is that the gold film is too thick, the loss will be significantly reduced, so the electric field cannot penetrate through the gold layer. If the gold film is too thin, the plasma wave will be strongly radiated and damped. We can also notice that the loss spectrum of $t=45 \mathrm{~nm}$ has a sharper resonance peak, thus $t=45 \mathrm{~nm}$ might be an optimal gold film thickness.

Fig. 3(c) shows the loss spectra at different distances $H$ from the D-shaped section to the fiber core. The larger $H$ makes the gold film area larger, which leads to the increase of the real part of the effective index of SPP mode. It can be seen from Fig. 3(c) that the resonance wavelength has a tendency to red shift with the increase of $H$. It is mainly because the effective refractive index of SPP mode increases. The loss spectrum with $H=3.5 \mu \mathrm{m}$ has a sharper resonance peak, so $H=3.5 \mu \mathrm{m}$ is the best profile height. Fig. 3(d) shows the impact of the $\Lambda$ on fiber sensing performance. The increase of 
hole-to-hole pitch $\Lambda$ leads to the decrease of gold film area, which is opposite to that of $H$. It can be seen from Fig. 3(d) that with the increase of $\Lambda$, there is blue shift in the resonance wavelength. Meanwhile, with the increase of $\Lambda$, the peak value and FWHM of the loss peak decrease. Thus $\Lambda=3.1 \mu \mathrm{m}$ might be an optimal hole-to-hole pitch.
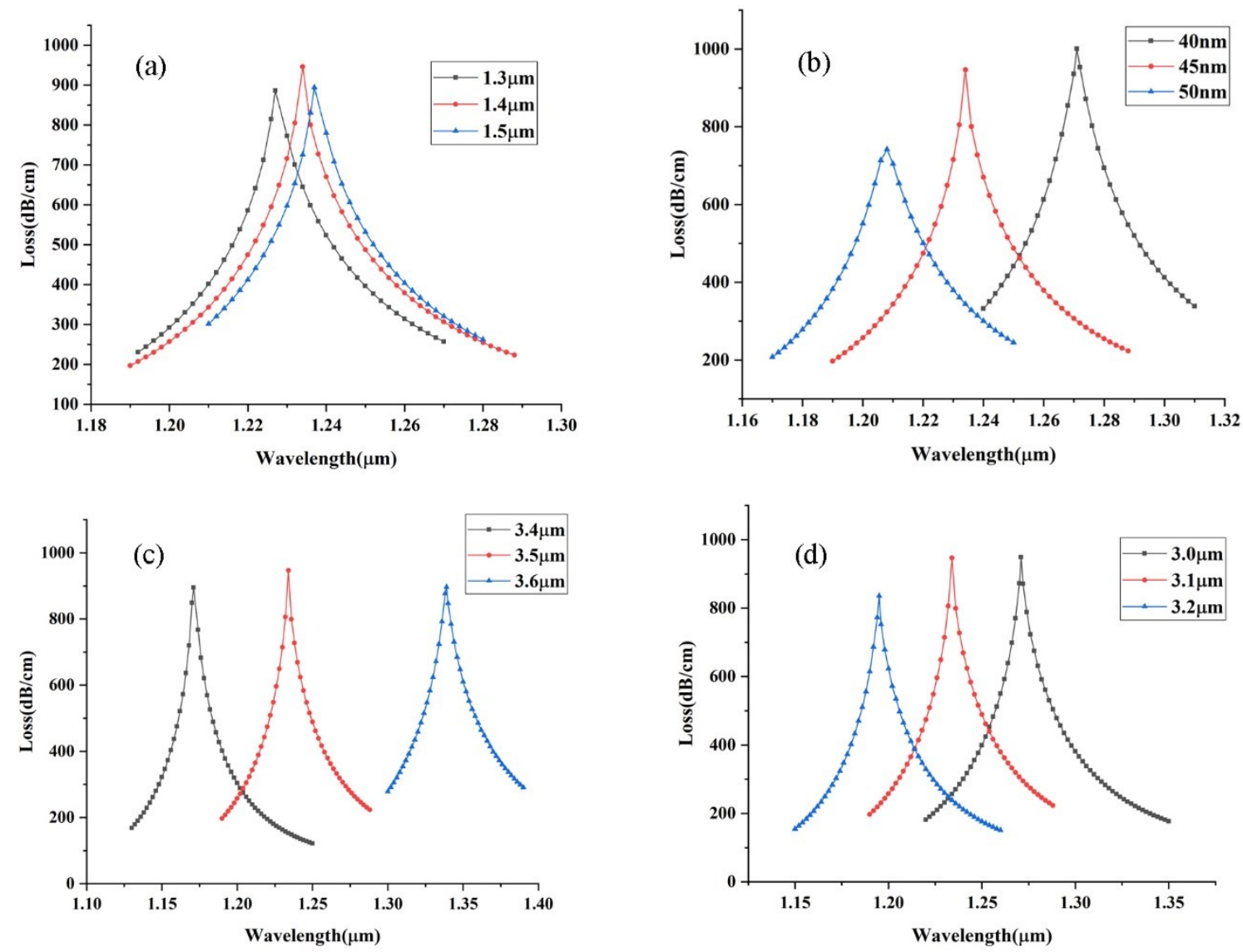

Fig. 3. Loss curves of the proposed SPR-PCF sensor for different hole diameters $d$ (a), different gold film thickness $t$ (b), different D-section distance (c) and different pitch size $\Lambda$ (d).

In the case of optimal structure parameters, we test the refractive index detection range and sensitivity of the sensor. Fig. 4(a) shows the loss curves of the sensor for different RIs of analyte. With the increase of the RI of the analyte, the resonance wavelength generates red shift. Fig. 4(b) shows the change of resonance wavelength when the refractive index of analyte changes from 1.26 to 1.38 . Outside the detection range, the energy of the fiber core will leak to the cladding, resulting in the increase of FWHM, which is not suitable for sensing. The interpolation table shows the polynomial fitting results, and the slope of the fitting curve represents the sensitivity of our proposed sensor. The adjusted R-square value of this fitting is 0.99992 , which indicates that the fitting is consistent. Tab.1. shows the comparison of the present work with the earlier published works. 

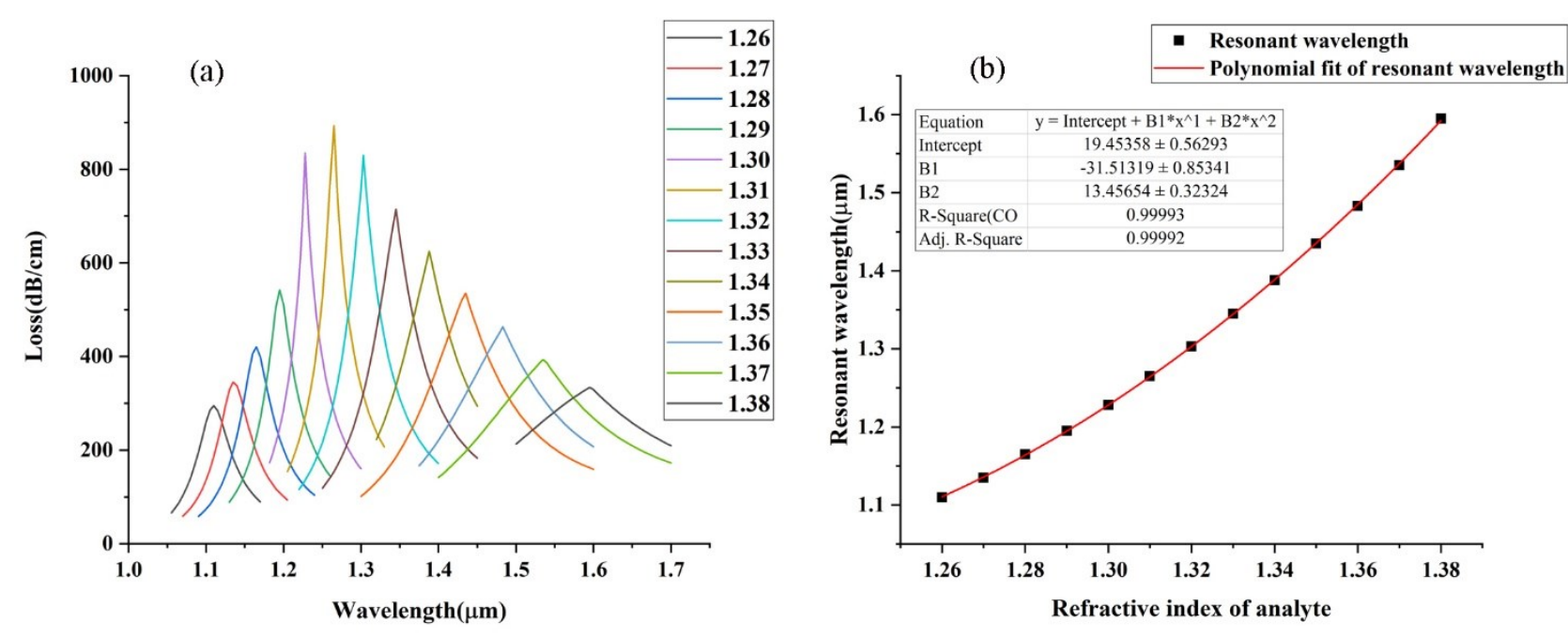

Fig. 4. Loss curves of the proposed SPR-PCF sensor for different analyte RI (a) and Variations of resonance wavelength with analyte RI (b).

Tab.1. Comparison of the reported SPR sensors

\begin{tabular}{ccc}
\hline Reference & Refractive index detection range & Sensitivity (nm/RIU) \\
\hline$[2]$ & $1.33-1.43$ & $2150 \mathrm{~nm} / \mathrm{RIU}$ \\
{$[15]$} & $1.20-1.29$ & $11055 \mathrm{~nm} / \mathrm{RIU}$ \\
{$[16]$} & $1.33-1.42$ & $11000 \mathrm{~nm} / \mathrm{RIU}$ \\
{$[17]$} & $1.23-1.29$ & $11000 \mathrm{~nm} / \mathrm{RIU}$ \\
This work & $1.26-1.38$ & $5626.86 \mathrm{~nm} / \mathrm{RIU}$ \\
\hline
\end{tabular}

\section{Conclusion}

In this paper, a novel D-type PCF sensor based on simple structure is proposed, and its numerical study is carried out. The sensor structure is a PCF based on two layers of regular hexagonal pores. The SPR effect can be triggered and the resonance effect can be enhanced by coating a thin gold film on the open air hole. The simulation results show that the maximum sensitivity is $5626.86 \mathrm{~nm} / \mathrm{RIU}$ and the resolution is $1.78 \times 10^{-5} \mathrm{RIU}$ in the refractive index detection range of $1.26-$ 1.38. The sensor uses PCF composed of pores with the same diameter, which simplifies the manufacturing process of the sensor. Meanwhile, the measurement of analytes with low refractive index (Less than the refractive index of water) and 
high refractive index (Greater than the refractive index of water) can be realized. The wide RI range enables the sensor to detect a variety of liquid refractive index, which has a broad application prospect.

\section{Acknowledgments}

This work was supported by key project of Tianjin Natural Science Foundation (No.20JCZDJC00500) and Tianjin Postgraduate Research and Innovation (No. 2020YJSS009).

\section{Declaration of Competing Interest}

The authors declare that they have not known competing financial interests or personal relationships that could have appeared to influence the work reported in this paper.

\section{References}

[1] E. Kretschmann, H. Raether. Notizen: Radiative Decay of Non Radiative Surface Plasmons Excited by Light[J]. Zeitschrift Für Naturforschung A, 1968, 23(12): 2135-2136.

[2] Z. Fan. Surface plasmon resonance refractive index sensor based on photonic crystal fiber covering nano-ring gold film[J]. Optical Fiber Technology, 2019, 50(JUL.):194-199.

[3] R. K. Gangwar, V. K. Singh. Highly Sensitive Surface Plasmon Resonance Based D-Shaped Photonic Crystal Fiber Refractive Index Sensor[J]. Plasmonics, 2017, 12(5): 1-6.

[4] S. Wang, S. Li. Surface plasmon resonance sensor based on symmetrical side-polished dual-core photonic crystal fiber[J]. Optical fiber technology, 2019, 51(SEP.):96-100.

[5] C. Liu, J. W. Wang, et al. Surface plasmon resonance (SPR) infrared sensor based on D-shape photonic crystal fibers with ITO coatings[J]. Optics Communications,2020,464:1-8.

[6] E. Kretschmann. The determination of the optical constants of metals by excitation of surface plasmons[J]. Z. Physik, 1971,241(4): 313-324.

[7] A. Otto. Excitation of Nonradiative Surface Plasma Waves in Silver by the Method of Frustrated Total Reflection[J]. Z. Physik, 1968, 216(4): 398-410.

[8] W. J. H. Bender, R. E. Dessy, et al. Feasibility of a Chemical Microsensor Based on Surface Plasmon Resonance on Fiber Optics Modified by Multilayer Vapor Deposition[J]. Analytical Chemistry, 1994, 66(7):963-970.

[9] R. Otupiri, E. K. Akowuah, S. Haxha. Multi-channel SPR biosensor based on PCF for multi-analyte sensing applications[J]. Optics Express, 2015, 23(12):15716-15727.

[10] R. Zakaria, W. Kam, et al. Fabrication and simulation studies on D-shaped optical fiber sensor via surface plasmon resonance[J]. Journal of Modern Optics, 2017:1-7.

[11] B. Shuai, L. Xia, and D. Liu, Coexistence of positive and negative refractive index sensitivity in the liquid-core photonic crystal fiber based plasmonic sensor[J]. Optics Express. 2012, 20(23): 25858-25866.

[12] C. D. Liang, C. Y. Xian, et al. Hollow-fiber-based surface plasmon resonance sensor with large refractive index detection range and high linearity[J]. Applied Optics, 2017, 56(36):9907-9912. 
[13] A. A. Rifat, H. Firoz, et al. Highly sensitive selectively coated photonic crystal fiber-based plasmonic sensor[J]. Optics letters, 2018, 43(4): 891-894.

[14] C. Liu, L. Yang, et al. Mid-infrared surface plasmon resonance sensor based on photonic crystal fibers[J]. Optics Express, 2017, 25(13):14227-14237.

[15] X. Chen, L. Xia, C. Li. Surface plasmon resonance sensor based on a novel D-shaped Photonic Crystal Fiber for low refractive index detection[J]. IEEE Photonics Journal, 2018, 10(1):1-9.

[16] A. K. Pathak, V. K. Singh. SPR Based Optical Fiber Refractive Index Sensor Using Silver Nanowire Assisted CSMFC[J]. IEEE Photonics Technology Letters, 2020, 32(8):465-468.

[17] S. Wang, X. H. Sun, et al. Surface plasmon resonance sensor based on D-shaped Hi-Bi photonic crystal fiber[J]. Optics Communications, 2020,467: 1-6.

[18] J. Boehm, F. Alexandre, et al. Chemical Deposition of Silver for the Fabrication of Surface Plasmon Microstructured Optical Fibre Sensors[J]. Plasmonics, 2011, 6(1):133-136.

[19] I. H. Malitson. Interspecimen Comparison of the Refractive Index of Fused Silica[J]. Journal of the Optical Society of America, 1965, 55:1205-1208.

[20] A. Vial, A. S. Grimault, et al. Improved analytical fit of gold dispersion: Application to the modeling of extinction spectra with a finite-difference time-domain method[J]. Physical Review B Condensed Matter, 2005, 71(8):85416.

[21] G. An, X. Hao, et al. D-shaped photonic crystal fiber refractive index sensor based on surface plasmon resonance[J]. Applied Optics, 2017, 56(24):6988-6992. 